\section{Cureus}

\title{
Idiopathic Urethral Stricture and Nephrogenic Diabetes Insipidus: The Odd Couple
}

\author{
Alessandro Maria Berton ${ }^{1}$, Nunzia Prencipe ${ }^{1}$ \\ 1. Endocrinology, Diabetology and Metabolism, University of Turin, Turin, ITA
}

$\square$ Corresponding author: Alessandro Maria Berton, alessandro.m.berton@gmail.com Disclosures can be found in Additional Information at the end of the article

\section{Abstract}

Nephrogenic diabetes insipidus (NDI) is one of the principal defects leading to polyuriapolydipsia syndrome (PPS). In the absence of other evident causes (drug interaction, electrolytic disorders or inherited disease), obstructive uropathy is the most likely aetiology. Direct arginine vasopressin (AVP) assessment during water deprivation test (WDT) remains the gold standard in PPS differential diagnosis despite well characterised limitations in this procedure. A new WDT method using copeptin as reliable surrogate of AVP is proposed. This case represents the first report of an NDI due to idiopathic urethral stricture in an adult and it would like to be explicative of the importance of a correct differential diagnosis of PPS and of the risk related to a prolonged WDT procedure in a frail patient. A 48-year-old male patient presenting with polyuria and polydipsia lasting one month was diagnosed with NDI. Copeptin values were clearly elevated both at baseline and after osmotic stimulus. WDT was complicated by development of acute kidney injury. Abdomen ultrasound demonstrated bilateral hydronephrosis, trabeculated bladder and a residual urine volume of $819 \mathrm{cc}$, in presence of normal kidney size and prostatic gland. A cysto-urethrography showed a sub-stenosis of $35 \mathrm{~mm}$ involving the membranous urethral tract. The patient underwent to balloon dilatation and urethrotomy with complete restitutio ad integrum. In our knowledge, this is the first report of idiopathic urethral stricture complicated by NDI in adult. PPS workup requires a global medical evaluation by an endocrinologist. In the suspicion of NDI, urinary tract obstruction should be considered. WDT remains a cornerstone in the differential diagnosis of PPS and the availability of biomarkers including copeptin may simplify the diagnostic process.

Categories: Endocrinology/Diabetes/Metabolism, Urology, Nephrology

Keywords: stenosis, polyuria, polydipsia, deprivation, aquaporin, copeptin

Received 05/08/2019

Review began 05/10/2019

Review ended 06/21/2019

Published 07/04/2019

\section{(C) Copyright 2019}

Berton et al. This is an open access article distributed under the terms of the Creative Commons Attribution License CC-BY 3.0., which permits unrestricted use, distribution, and reproduction in any medium, provided the original author and source are credited.

\section{Introduction}

Nephrogenic diabetes insipidus (NDI) is one of the principal defects leading to polyuriapolydipsia syndrome (PPS), together with central diabetes insipidus and primary polydipsia (PP). NDI results from failure of the kidneys to concentrate urine due to a renal insensitivity to the action of the antidiuretic hormone arginine vasopressin (AVP) [1]. Both primary and secondary forms of the disease have been described: primary NDI is principally due to an inherited mutation of vasopressin V2 receptor (AVPR2) or aquaporin-2 (AQP2) genes; whereas secondary NDI is due to lithium treatment, hypercalcemia, hypercalciuria, hypokalemia or obstructive uropathy. In obstructive uropathy, NDI results from suppression of AQP2 expression mediated by increased hydrostatic pressure [2]; missing this diagnosis can lead to end-stage renal disease [3]. Direct AVP assessment during water deprivation test (WDT) is considered the gold standard in PPS differential diagnosis [4]. However, mainly because of the 


\section{Cureus}

large preanalytical variability of AVP assay, a new WDT method has been proposed using copeptin as reliable surrogate of AVP [5,6]. The following case report represents the first description of an NDI due to idiopathic urethral stricture in an adult. It is also an example of acute kidney injury as a possible WDT side effect.

\section{Case Presentation}

A 48-year-old smoker male patient was admitted to our Endocrinology Division for polyuria, mostly nycturia and polydipsia lasting one month. The patient also had urgency urinary incontinence, but not voiding difficulty, temperature or stranguria. Past medical history revealed an untreated atrial flutter (CHADs-VASC 0) and pacemaker implant for sick sinus syndrome; but no history of traumatic brain injury was detected. Family history revealed no significant diseases. Physical examination showed a first-degree obesity (Body Mass Index 32.7 $\mathrm{kg} / \mathrm{m}^{2}$ ), mild pretibial bilateral oedema and high heart rate (110 beats per minute). Head, neck, lymph nodes and chest examinations showed no abnormalities. Laboratory data indicated the patient had normal serum electrolyte levels $\left(\mathrm{Na}^{+} 141 \mathrm{mmol} / \mathrm{L}, \mathrm{K}^{+} 3.6 \mathrm{mmol} / \mathrm{L}, \mathrm{Ca}^{++} 2.28\right.$ $\mathrm{mmol} / \mathrm{L}$ ), normal glycometabolic profile (fasting glucose $79 \mathrm{mg} / \mathrm{dl}$, HbA1c $47 \mathrm{mmol} / \mathrm{mol}$ ), mild elevation of NT-pro-BNP (1018 pg/ml) of uncertain clinical relevance, normal PSA level (0.38 $\mathrm{ng} / \mathrm{ml}$ ) and progressive increase in serum creatinine (1.45 versus $0.8 \mathrm{mg} / \mathrm{dl})$ in the last year. The hydric balance showed a water intake almost equal to output (6-9 liters per day). Due to suspected diabetes insipidus, plasmatic (p-Osm) and urinary (u-Osm) osmolality evaluations were performed. The analyses showed improperly lower u-Osm levels $(121 \mathrm{mOsm} / \mathrm{kg})$, in presence of mild-elevated p-Osm $(299 \mathrm{mOsm} / \mathrm{kg}$ ) with reduced $\mathrm{u}$-Osm/p-Osm ratio (0.4) and urine specific gravity (1.007), in the absence of glycosuria or proteinuria. Thyroid and adrenal deficits were excluded (TSH $2.9 \mu \mathrm{UI} / \mathrm{ml}$, fT4 $11.3 \mathrm{pg} / \mathrm{ml}$, cortisol $148.2 \mu \mathrm{g} / \mathrm{L}$, ACTH $40 \mathrm{pg} / \mathrm{ml}$ ). In presence of transient psychiatric agitation symptoms, computed tomography scan was performed to exclude frontal cerebral lesions, and no evidence of encephalic, hypothalamic or pituitary lesions was found.

Following our local clinical standard practice, an indirect WDT followed by a D-amino Darginine vasopressin (DDAVP) test (4 $\mu$ g s.c.) was performed (Tables 1,2$)$.

\begin{tabular}{|c|c|c|c|c|c|c|c|}
\hline Time & 0 AM & $8 \mathrm{AM}$ & 9 AM & 10 AM & 11 AM & 12 AM & 1 PM \\
\hline u-Osm (mOsm/kg) & 143 & 146 & 147 & 154 & 157 & 164 & 172 \\
\hline p-Osm (mOsm/kg) & 286 & 297 & 297 & 298 & 297 & 298 & 299 \\
\hline $\mathrm{Na}^{+}(\mathrm{mmol} / \mathrm{L})$ & 138 & 145 & 144 & 146 & 146 & 145 & 147 \\
\hline Partial diuresis (cc) & 0 & +900 & +150 & +150 & +50 & +150 & +400 \\
\hline Copeptın (pmol/L) & 35.4 & NA & NA & NA & NA & NA & 57.3 \\
\hline
\end{tabular}

\section{TABLE 1: Water deprivation test.}

u-Osm: Urine osmolality; p-Osm: Plasma osmolality; Na+: Serum sodium; NA: Not available; AM: Ante meridiem; PM: Post-meridiem. 


\section{Cureus}

\begin{tabular}{|c|c|c|c|}
\hline Time after DDAVP administration & $0^{\prime}$ & 60 ' & 120 ' \\
\hline u-Osm (mOsm/kg) & 195 & 196 & 212 \\
\hline Partial diuresis (cc) & 0 & +200 & +100 \\
\hline
\end{tabular}

\section{TABLE 2: DDAVP test.}

DDAVP: D-amino D-arginine vasopressin; u-Osm: Urine osmolality.

According to our local protocol, dehydration test was stopped when serum sodium reached 147 $\mathrm{mmol} / \mathrm{L}$ and an effective osmotic stimulus was obtained (p-Osm 299 versus $286 \mathrm{mOsm} / \mathrm{kg}$ and

$\mathrm{Na}^{+} 147$ versus $138 \mathrm{mmol} / \mathrm{L}$; peak versus baseline, respectively) in the absence of significant $\mathrm{u}^{-}$ Osm variations ( $\mathrm{u}$-Osm 172 versus $143 \mathrm{mOsm} / \mathrm{kg}$; peak versus baseline); thus confirming diabetes insipidus diagnosis. In addition, a lack of response to DDAVP test was observed (u-Osm 195 versus $212 \mathrm{mOsm} / \mathrm{kg}$; basal and post-DDAVP stimulus), suggesting complete NDI. Copeptin values were clearly elevated both at baseline and after osmotic stimulus ( 35.4 and $57.3 \mathrm{pmol} / \mathrm{L}$, respectively). According to nephrological indication, infiltrating disorders (sarcoidosis, amyloidosis, multiple myeloma, Sjogren’s disease) were excluded.

In the following days, serum creatinine levels abruptly increased $(3.2 \mathrm{mg} / \mathrm{dl}$; estimated Glomerular Filtration Rate sec. CKD-EPI $22 \mathrm{ml} / \mathrm{min} / 1.73 \mathrm{~m}^{2}$ ) suggesting acute kidney injury (AKI), although appropriate hydration per os was guaranteed. The abdomen ultrasound demonstrated bilateral first degree hydronephrosis, trabeculated bladder and a residual urine volume of $819 \mathrm{cc}$, in presence of normal kidney size with preserved cortical thickness and normal prostatic gland with central hyperechogenicity. No radiological signs of urinary stones were detected (Figure 1).

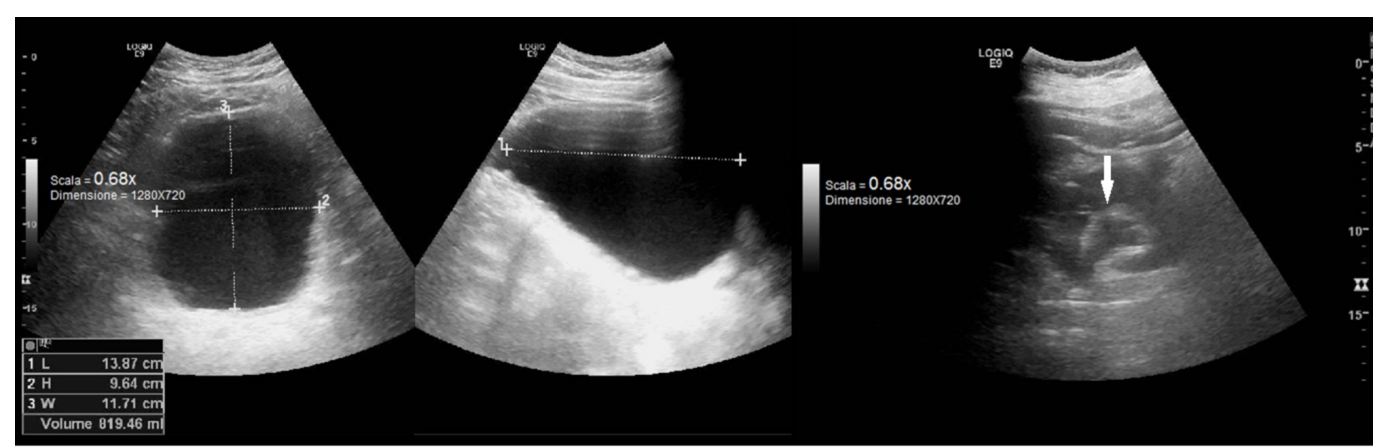

FIGURE 1: Abdomen ultrasound showing residual urine volume and first degree hydronephrosis (white arrow).

L: Length; H: Height; W: Width.

These radiological findings allowed a better definition of the PPS, in which an overflow incontinence was likely hidden by the polyuric state. Therefore, a retrograde cysto- 


\section{Cureus}

urethrography was performed, highlighting a sub-stenosis of $35 \mathrm{~mm}$ involving the membranous urethra tract, with urologic indication for urethrocystoscopy and balloon dilatation. Eventually stricture was resolved with urethrotomy and subsequent long-term urethral catheterization (Figure 2).

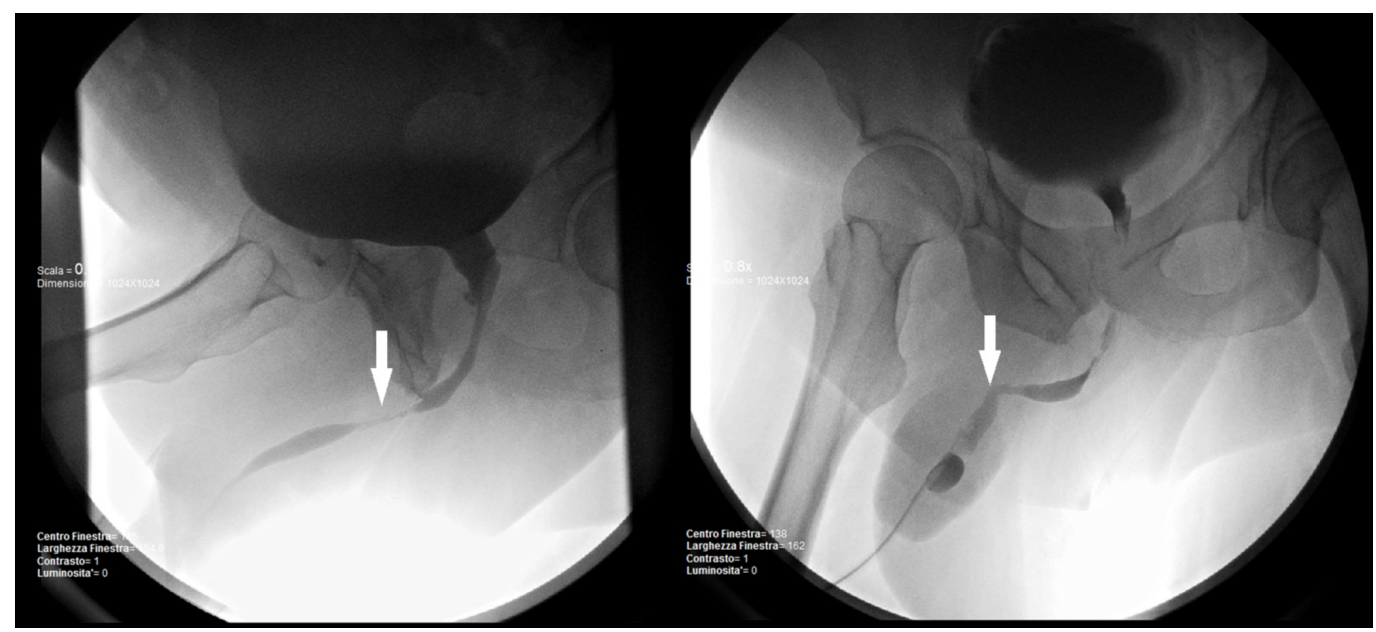

FIGURE 2: Retrograde cysto-urethrography showing the location, length, and severity of the stricture pre- and postdilatation procedure (white arrow).

One month after catheter removal, a urodynamic study confirmed the resolution of the urinary obstruction and an abdominal ultrasound showed normal residual urine volume in the absence of hydronephrosis. Laboratory analyses demonstrated a restored hydro-electrolytic balance $\left(\mathrm{Na}^{+} 140\right.$ mmol/L, p-Osm 291 mOsm/kg, u-Osm 377 mOsm/kg, urine specific gravity 1.010, copeptin $15.9 \mathrm{pmol} / \mathrm{L}$ ) and a normalization of the renal function (creatinine $0.89 \mathrm{mg} / \mathrm{dl}$, estimated Glomerular Filtration Rate sec. CKD-EPI $100.4 \mathrm{ml} / \mathrm{min} / 1.73 \mathrm{~m}^{2}$ ) along with remission of the urinary symptoms and normalization of the water input.

\section{Discussion}

In the absence of other evident causes (drug interaction, electrolytic disorders or inherited disease), obstructive uropathy is the most likely NDI aetiology and a comprehensive urinary tract evaluation is mandatory. Urethral strictures (idiopathic or related to endoscopic procedure, traumatism or inflammatory disease) are not an infrequent cause of obstructive disease (incidence 200-1,200 cases per 100,000 individuals, increasing in people aged $\geqslant 55$ years) and majority of them are anterior (92.2\%) involving the bulbar urethra (46.9\%) [7]. In our case, secondary forms have been excluded which to our knowledge represents the first report of idiopathic urethral stricture complicated by NDI in adult.

WDT is the essential tool in differential diagnosis flowchart of PPS and is used to identify the aetiology of the syndrome (primary polydipsia, central or nephrogenic diabetes insipidus), but as known, it requires hospitalization, it is time-consuming (more than 20 hours may be needed), often hard for the patient to tolerate and can lead to severe clinical consequences. Indirect WDT, based on u-Osm variation induced by dehydration first and then by DDAVP administration, is characterized by poor diagnostic accuracy [5]. Direct WDT with AVP measurement is not routinely used in endocrinological practice because of its large preanalytical variability [8]. Copeptin, the C-terminal portion of the AVP precursor, is a stable, surrogate marker that can replace AVP testing. Copeptin is secreted in equimolar levels to AVP 
by neurosecretory granules of neurohypophysis [9]. Two recent clinical trials have proposed a new WDT method using copeptin as reliable surrogate of AVP $[5,6]$. This test is demonstrated to be more accurate and, crucially, dehydration would no longer be necessary in NDI identification because basal copeptin value $>21.4 \mathrm{pmol} / \mathrm{L}$ detects $100 \%$ of polyuric patients affected by AVP insensitivity [6].

In our patient, AKI developed immediately after WDT, suggesting the convenience of a shorter dehydration period or a different diagnostic test. In fact, AKI is a rare but severe complication, which may even require to dialysis in some cases [3]. In this context for example, an initial correct definition of urinary symptoms (overflow incontinence vs urinary urgency), together with basal copeptin determination could already be suggestive for NDI.

Our experience is finally consistent with the recent evidence that copeptin is a valid alternative to WDT and may be especially useful in managing frail patients (e.g., cardiac disease, chronic renal failure, incontinence, psychiatric disorders) and in the elderly. Frequently in these patients prolonged dehydration is not only difficult to be performed, but also unsafe.

\section{Conclusions}

All polyuric-polydipsic patients require a global medical evaluation and the endocrinologist plays the main role in their management. In the suspicion of NDI, all possible obstructive urological causes should be considered in order to be surgical treated preventing further kidney damage. Although direct or indirect WDT remains the cornerstone of the differential diagnosis of the syndrome, a prolonged dehydration could be dangerous in severe polyuric patients. The availability of copeptin may improve and simplify the diagnostic process in these patients, avoiding the WDT practice in NDI.

\section{Additional Information}

\section{Disclosures}

Human subjects: Consent was obtained by all participants in this study. Conflicts of interest: In compliance with the ICMJE uniform disclosure form, all authors declare the following: Payment/services info: All authors have declared that no financial support was received from any organization for the submitted work. Financial relationships: All authors have declared that they have no financial relationships at present or within the previous three years with any organizations that might have an interest in the submitted work. Other relationships: All authors have declared that there are no other relationships or activities that could appear to have influenced the submitted work.

\section{Acknowledgements}

The authors thank Silvia Grottoli and Ezio Ghigo for their scientific contribution and constructive recommendations on this project.

\section{References}

1. Bockenhauer D, Bichet DG: Pathophysiology, diagnosis and management of nephrogenic diabetes insipidus. Nat Rev Nephrol. 2015, 11:576-588. 10.1038/nrneph.2015.89

2. Li C, Wang W, Kwon TH, et al.: Downregulation of AQP1, -2 , and -3 after ureteral obstruction is associated with a long-term urine-concentrating defect. Am J Physiol Renal Physiol. 2001, 281:163-171. 10.1152/ajprenal.2001.281.1.F163

3. Zender HO, Ruedin P, Moser F, Bolle JF, Leski M: Traumatic rupture of the urinary tract in a patient presenting nephrogenic diabetes insipidus associated with hydronephrosis and chronic renal failure: case report and review of the literature. Clin Nephrol. 1992, 38:196-202. 


\section{Cureus}

4. Fenske W, Allolio B: Current state and future perspectives in the diagnosis of diabetes insipidus: a clinical review. J Clin Endocrinol Metab. 2012, 97:3426-3437. 10.1210/jc.20121981

5. Fenske W, Quinkler M, Lorenz D, et al.: Copeptin in the differential diagnosis of the polydipsia-polyuria syndrome-revisiting the direct and indirect water deprivation tests. J Clin Endocrinol Metab. 2011, 96:1506-1515. 10.1210/jc.2010-2345

6. Timper K, Fenske W, Kühn F, et al.: Diagnostic accuracy of copeptin in the differential diagnosis of the polyuria-polydipsia syndrome: a prospective multicenter study. J Clin Endocrinol Metab. 2015, 100:2268-2274. 10.1210/jc.2014-4507

7. Hampson LA, McAninch JW, Breyer BN: Male urethral strictures and their management . Nat Rev Urol. 2014, 11:43-50. 10.1038/nrurol.2013.275

8. Heida JE, Boesten LSM, Ettema EM, Muller Kobold AC, Franssen CFM, Gansevoort RT, Zittema D: Comparison of ex vivo stability of copeptin and vasopressin . Clin Chem Lab Med. 2017, 55:984-992. 10.1515/cclm-2016-0559

9. Morgenthaler NG, Struck J, Alonso C, Bergmann A: Assay for the measurement of copeptin, a stable peptide derived from the precursor of vasopressin. Clin Chem. 2006, 52:112-119. 10.1373/clinchem.2005.060038 